\title{
Distorsiones Cognitivas en Agresores de Pareja: Análisis de una Herramienta de Evaluación
}

\author{
Cognitive Distortions Among Partner-Violent Men: Analyzing an Assessment Tool
}

\author{
Ismael Loinaz \\ Universidad del País Vasco UPV/EHU, España
}

(Rec: 10 mayo 2013 / Acept: 12 diciembre 2013)

\begin{abstract}
Resumen
Las distorsiones cognitivas influyen en el desarrollo y mantenimiento de la violencia. En agresores de pareja, la negación del problema y la culpabilización de la víctima son especialmente prevalentes, promoviendo una menor asunción de responsabilidades. Este trabajo revisa las propiedades psicométricas de un inventario diseñado para la evaluación de pensamientos distorsionados sobre la mujer y la violencia. Se analiza su aplicación en 180 agresores en prisión, así como los resultados de 11 estudios previos, comparando dos formatos de corrección (verdadero/falso y Likert/factorial). El análisis factorial permitió eliminar los ítems 7, 8, 19 y 28 y agrupar el contenido en 4 factores. El ítem 27 se eliminó por considerarlo erróneo. Se detectaron limitaciones de la herramienta, como una poca sensibilidad al cambio terapéutico y baja capacidad discriminativa entre distintas muestras. Se discuten las precauciones a tener en cuenta a la hora de utilizar la herramienta en el ámbito profesional.

Palabras clave: distorsiones cognitivas; violencia; agresores de pareja; propiedades psicométricas
\end{abstract}

Abstract

Cognitive distortions are related to the development and maintenance of violent behavior. Among partnerviolent men the denial of the problem, and victim blaming are especially prevalent, and reduce accountability. This paper reviews the psychometric properties of an inventory designed to assess distortions about women and violence justification. Its application in 180 offenders in prison is analyzed, as well as the results of 11 previous studies, comparing two correction options (true/false and Likert/factorial). Factor analysis allowed to remove items 7, 8, 19 and 28 due to their low statistical significance, and to group them in four factors. Item 27 was removed because is considered to be wrong. Some limitations were detected, as can be a low sensitivity to therapeutic change, and a low capacity to discriminate between different samples. Precautions to be taken when using the tool in the professional field are discussed.

Key words: cognitive distortions; violence; partner-violent men; psychometric properties 


\section{Introducción}

En el desarrollo de la conducta violenta influyen variables biológicas, psicológicas, sociológicas y contextuales (Shaver y Mikulincer, 2011). Entre las variables psicológicas, las más mencionadas son los trastornos de la personalidad, las adicciones, la baja empatía y las distorsiones cognitivas. Estas últimas son especialmente frecuentes en los programas de intervención para delincuentes.

Las distorsiones cognitivas son formas erróneas de interpretar la realidad. Están presentes en distintos tipos de trastornos mentales (como la depresión, los trastornos alimentarios o el trastorno psicótico entre otros) y su descripción es habitual en delincuentes y personas violentas (Gannon, Ward, Beech y Fisher, 2007). En el ámbito criminológico, pueden considerarse cogniciones mantenedoras de la conducta cuyo objetivo es eximir la responsabilidad y las consecuencias de una conducta socialmente reprobable. El delito se racionaliza, principalmente mediante la minimización, la negación o la atribución de culpa a los demás.

En uno de los primeros trabajos sobre la temática en el contexto penitenciario, Henderson y Hewstone (1984) pusieron de manifiesto cómo las explicaciones de la conducta violenta eran externas con mayor frecuencia (culpabilizando a la víctima o a situaciones ajenas) y más de tipo justificativo. Pese a su frecuencia en el ámbito de estudio, su uso es poco claro y la terminología empleada muy diversa: procesos cognitivos, actitudes, creencias, pensamientos concretos en situaciones específicas, justificaciones posthoc, etc. (Helmus, Hanson, Babchishin y Mann, 2013). Esta variedad de conceptos hace que tan solo algunos de ellos estén realmente relacionados con el desarrollo de conductas delictivas y que, por ello, los resultados sean dispares.

En la evaluación de la violencia contra la pareja (VCP), la minimización o la negación por parte de ambos miembros es una complicación habitual (Rathus y Feindler, 2004). Los agresores son especialmente propensos a minimizar su existencia o impacto, y las víctimas a negar lo ocurrido o asumir la culpa. La ausencia de responsabilidad, la atribución de culpa a factores externos como forma de justificarse (estrés, alcohol, características de la víctima), la minimización de lo ocurrido o su negación son aspectos habituales en agresores de pareja (Bowen, 2011; Lila, Gracia y Herrero, 2012; Lila, Herrero y Gracia, 2008a). Sin embargo, existen pocos datos sobre su presencia o influencia previa al delito (Bowen, 2011) $\mathrm{y}$, en realidad, estos sesgos son analizados como actitudes post-hoc (justificaciones de lo ocurrido), por lo que puede ser inadecuado tomarlos como objetivo de la intervención (Novo, Fariña, Seijo y Arce, 2012).
El estilo de respuesta socialmente deseable, la atribución de la culpa al otro miembro de la pareja y la negación o minimización de lo ocurrido están presentes tanto en hombres como en mujeres condenados por VCP (Henning, Jones y Holdford, 2005). Incluso en el caso de la población general, como los estudiantes universitarios, la minimización y la culpabilización de la pareja se relaciona con el autoinforme de violencia (Scott y Straus, 2007). Algunos sesgos, como la culpabilización de la víctima, no solo se dan en los agresores sino que, en ocasiones, forman parte de las propias actitudes de la sociedad en conjunto (ValorSegura, Expósito y Moya, 2008, 2011).

Las distorsiones cognitivas contribuyen a la VCP al promover que el agresor atienda selectivamente a determinados aspectos, tergiverse o distorsione situaciones, incrementando la probabilidad de sentir ira y reaccionar con agresiones (Eckhardt y Kassinove, 1998; HoltzworthMunroe y Hutchinson, 1993). Dentro del ámbito de estudio, es especialmente frecuente el denominado error fundamental de atribución. Este tipo de pensamiento hace que conductas propias sean achacadas a factores externos, temporales y específicos, mientras que aquellas ajenas son atribuidas a factores internos, permanentes y generalizados (Maruna y Mann, 2006). En el caso de los agresores, la conducta de la pareja es atribuida a características personales (ella es asi) mientras que la propia se atribuye a factores externos o situacionales (estaba muy estresado o había bebido). Sin embargo, se afirma que este tipo de excusas post-hoc son comunes fuera del ámbito delictivo, por lo que atribuirles características criminógenas sería una falacia.

Dutton (1986) analizó la descripción de la última agresión en 75 hombres con historial de violencia repetida contra su pareja, poniendo de manifiesto que el $21 \%$ aceptaba que el hecho estaba mal, pero se excusaba no considerándolo responsabilidad suya. El 79\% restante aceptaba su responsabilidad, pero justificaba los hechos echándole la culpa a la víctima o restando valor a lo ocurrido. Estos últimos, además, eran más propensos a minimizar la frecuencia, gravedad o consecuencias de su agresión. El patrón de distorsión o respuesta socialmente deseable es diferente según el tipo de muestra, pudiendo ser mayor en los casos de agresores que acuden voluntariamente a los servicios de atención (Dutton y Starzomski, 1994), diferencia confirmada por Bowen y Gilchrist (2004) quienes encontraron un locus de control interno (asunción de culpa) significativamente mayor en los agresores voluntarios.

Wallach y Sela (2008) han revisado distintos tipos de estilo de atribución que influyen en la interpretación del origen de los problemas y la responsabilidad en su resolución. 
En general, los agresores negarían la comisión del acto o su responsabilidad, achacarían la culpa a factores externos o justificarían lo ocurrido, en todo caso, eliminando la necesidad de buscar una solución. Los autores indican que estos estilos de atribución incrementan la hostilidad y el estrés, junto al riesgo de conducta violenta.

El análisis cualitativo de relatos de hombres que habían matado a su pareja indica que, también en estos casos, los agresores pueden negar, justificar o racionalizar lo ocurrido parar evitar su responsabilidad, recurriendo a historias de victimización en la infancia (o en la edad adulta a manos de la policía o el sistema) o dirigiendo la culpa hacia la víctima (Dobash y Dobash, 2011), algo que según los autores del estudio influye en la incapacidad para sentir remordimiento o empatizar con la víctima.

El estudio de las distorsiones cognitivas es especialmente prevalente en el ámbito de la violencia sexual (ver por ejemplo Beech, Craig y Browne, 2009; Boer, Eher, Craig, Miner y Pfäfflin, 2011). El efecto de estas cogniciones, en especial la atribución de culpa, junto a interpretaciones, mitos y estereotipos pueden promover este tipo de violencia (Trujano y Raich, 2000). Sin embargo, se afirma que el concepto de distorsión cognitiva es excesivamente heterogéneo, poco preciso, y con poco fundamento experimental (Ó Ciardha y Ward, 2013). Asimismo, un amplio meta-análisis reciente indica que la influencia de las distorsiones en la reincidencia sexual, aunque consistente entre estudios, es pequeña $(d=0.22)$ (Helmus et al., 2013). Pese a ello, este meta-análisis concluye que: 1) las actitudes que apoyan la violencia sexual predicen la reincidencia; 2) tiene la misma capacidad predictiva la evaluación pre o post tratamiento; 3) la predicción es mejor cuando se asocia a tipologías de agresores; y 4) la predicción es mejor en agresores pedófilos.

Tal como adelantábamos, las distorsiones cognitivas son un objetivo habitual de los programas de intervención para distintos tipos de agresores. Modificar patrones cognitivos es un componente central de las técnicas cognitivo-conductuales y estas aproximaciones son predominantes (y las más avaladas) en el tratamiento de delincuentes. La asunción de responsabilidad y el abordaje de las distorsiones son objetivos centrales de la mayoría de los programas para agresores de pareja (Bowen, 2011; Lila et al., 2012) y su modificación se considera un criterio de eficacia de los mismos (Lila, Oliver, Galiana y Gracia, 2013). Es tal su importancia que existen programas centrados exclusivamente en su tratamiento. Estos programas de Confrontación de Creencias, con una extensión de 12 sesiones, han demostrado una eficacia similar a los programas centrados en la Gestión de la Ira (Russell, 2002).
Pese a la relevancia teórica de este tipo de variables y su inclusión como objetivos prioritarios de muchos programas de intervención, trabajos como el de Henning y Holdford (2006) no han encontrado una relación significativa entre la minimización, la negación y la culpabilización de la víctima con la reincidencia.

Aunque se consideren de interés en el ámbito criminológico, pocos estudios han analizado empíricamente las distorsiones cognitivas de los agresores de pareja o, más en concreto, la eficacia de las herramientas con las que afirmamos su presencia. Distintos autores ponen de manifiesto la necesidad de abordar este aspecto y diseñar herramientas útiles para su evaluación (Lila et al., 2012; Peters, 2008; Scott y Straus, 2007). El presente trabajo tiene como objetivo analizar las propiedades psicométricas de una escala destinada a la evaluación de pensamientos distorsionados relacionados con la violencia de pareja a partir de datos provenientes de su aplicación a agresores condenados por este delito. Junto a ello, se analiza su eficacia a través de once trabajos que han utilizado la herramienta en los 15 años transcurridos desde su creación.

\section{Método}

\section{Participantes}

La muestra estuvo compuesta por 180 agresores condenados a prisión en España, por un delito en el que la víctima fuese la pareja o expareja (131 del Centro Penitenciario Brians-2, Barcelona; 49 de Alhaurín de la Torre, Málaga). La edad media de los participantes fue de 38 años $(d t=8.37$; rango 22-61). El 78.7\% era español $(n=140)$. La mayoría $(61.5 \%$ ) tenía estudios secundarios (un $24.8 \%$ primarios, un $10.6 \%$ universitarios y un $3,1 \%$ sin estudios).

\section{Instrumentos}

Inventario de pensamientos distorsionados sobre la mujer y el uso de la violencia (IPDMV; Echeburúa y FernándezMontalvo, 1998). El IPDMV es un instrumento español diseñado para la evaluación de las distorsiones cognitivas en agresores de pareja. Su versión original está compuesta por 29 ítems repartidos en dos escalas: 13 ítems sobre roles sexuales e inferioridad de la mujer, y 16 sobre el uso de la violencia como medio aceptable para resolver conflictos. Tiene un formato de respuesta de verdadero o falso y se 
indica que cada respuesta afirmativa computa como una distorsión, siendo el resultado final el sumatorio de respuestas afirmativas. Desde su creación, no se han descrito sus propiedades psicométricas, aunque en un trabajo (Echeburúa, Sarasua, Zubizarreta y de Corral, 2009) se hace alusión a dos criterios sin indicar la procedencia del dato: consistencia interna (.87 para la escala Mujer y .94 para Violencia) y fiabilidad test-retest (.92 y .89 respectivamente).

Ferrer, Bosch, Ramis, Torres y Navarro (2006) propusieron una versión factorial del inventario. Los autores sustituyeron la respuesta dicotómica por una escala Likert de 4 puntos y eliminaron aquellos ítems que correlacionaban menos de .30 con la puntuación total (ítems 8, 19, 27, 28 y 29). La versión final constó de 24 ítems y presentó un alfa de Cronbach de .84. Por último, propusieron una corrección que agrupa los ítems en cuatro factores: 1) Aceptación del estereotipo tradicional y la misoginia; 2) Culpabilización de las mujeres víctimas del maltrato; 3) Aceptación de la violencia como forma adecuada para la solución de problemas; y 4) Minimización de la violencia contra las mujeres como problema y desculpabilización del maltratador.

\section{Procedimiento}

Todos los sujetos fueron evaluados en el contexto de una línea de investigación sobre agresores de pareja (ver Loinaz, 2014; Loinaz, Ortiz-Tallo y Ferragut, 2012; Loinaz, Ortiz-Tallo, Sánchez y Ferragut, 2011). Su participación fue voluntaria y previa firma del consentimiento informado. La evaluación se efectuó entre los años 2008 y 2010, y fue efectuada por el autor del estudio o por personal entrenado para tal objetivo (en el caso de Alhaurín de la Torre), en ambos casos personas ajenas a la institución penitenciaria (con ello se buscó la mayor sinceridad de los participantes al no afectar sus respuestas a la ejecución de la condena).

\section{Análisis de datos}

En primer lugar se analizaron las puntuaciones y las propiedades psicométricas del inventario en la muestra de agresores, comparando las dos propuestas de corrección disponibles: distribución de las respuestas, consistencia interna (alfa de Cronbach) y sensibilidad al cambio. En segundo lugar, se efectuó un análisis factorial exploratorio (método de extracción de componentes principales con rotación Varimax), analizando la posibilidad de eliminar aquellos ítems con cargas inferiores a .4 (Stevens, 2009), manteniendo aquellas $\geq .3$ si mejoraban la consistencia del factor. Por último, se realizó un análisis de los resultados obtenidos en estudios previos, calculando los tamaños del efecto ( $d$ de Cohen) en los casos en los que fuese posible.

\section{Resultados}

\section{Frecuencia de respuesta}

En la tabla 1 se muestra la distribución de respuesta a cada uno de los ítems. Como se puede observar, son especialmente prevalentes las respuestas afirmativas a los ítems 7,26 y 28 en más del $60 \%$, y 10, 11 y 26 en más del $50 \%$. 
Tabla 1. Distribución de las respuestas en formato original y escala Likert

\begin{tabular}{|c|c|c|c|c|c|}
\hline \multirow[b]{3}{*}{ Mujer } & \multicolumn{5}{|c|}{ Formato de respuesta } \\
\hline & \multirow{2}{*}{$\begin{array}{c}\text { Verdadero/ } \\
\text { Falso }\end{array}$} & \multicolumn{4}{|c|}{$\begin{array}{c}\text { Escala Likert } \\
\%\end{array}$} \\
\hline & & 1 & 2 & 3 & 4 \\
\hline 1. Las mujeres son inferiores a los hombres & 3.9 & 91.7 & 4.4 & 1.7 & 2.2 \\
\hline 2. Si el marido es el que aporta el dinero en casa, la mujer debe estar subordinada a él & 3.9 & 88.9 & 7.2 & 2.8 & 1.1 \\
\hline 3. El marido es el responsable de la familia, por lo que la mujer le debe obedecer & 6.7 & 86.7 & 6.7 & 4.4 & 2.2 \\
\hline 4. La mujer debe tener la comida y la cena a punto para cuando el marido vuelva a casa & 15.0 & 77.2 & 7.8 & 7.2 & 7.8 \\
\hline $\begin{array}{l}\text { 5. La obligación de una mujer es tener relaciones sexuales con su marido. aunque en ese } \\
\text { momento no le apetezca }\end{array}$ & 1.7 & 92.2 & 6.1 & 1.7 & 0.0 \\
\hline 6. Una mujer no debe llevar la contraria a su marido & 5.6 & 83.3 & 11.1 & 1.7 & 3.9 \\
\hline $\begin{array}{l}\text { 7. Una mujer que permanece conviviendo con un hombre violento debe tener un serio pro- } \\
\text { blema psicológico }\end{array}$ & 65.6 & 31.1 & 3.3 & 17.2 & 48.3 \\
\hline $\begin{array}{l}\text { 8. Para muchas mujeres, el maltrato por parte de sus maridos es una muestra de su preocu- } \\
\text { pación por ellas }\end{array}$ & 16.7 & 80.0 & 3.3 & 8.3 & 8.3 \\
\hline 9. Cuando un hombre pega a su mujer. ella ya sabrá por qué & 18.3 & 73.9 & 7.8 & 8.3 & 10 \\
\hline 10. Si las mujeres realmente quisieran, sabrían cómo prevenir nuevos episodios de violencia & 53.3 & 40 & 6.7 & 11.7 & 41.7 \\
\hline $\begin{array}{l}\text { 11. Muchas mujeres provocan deliberadamente a sus maridos para que éstos pierdan el } \\
\text { control y les golpeen }\end{array}$ & 51.7 & 34.3 & 13.9 & 15 & 36.7 \\
\hline 12. Si una mujer tiene dinero, no tiene por qué soportar una relación en la que existe violencia & 27.8 & 66.7 & 5.6 & 8.9 & 18.9 \\
\hline $\begin{array}{l}\text { 13. El hecho de que la mayoría de las mujeres no suela llamar a la policía cuando están siendo } \\
\text { maltratadas, prueba que quieren proteger a sus maridos }\end{array}$ & 36.7 & 50.0 & 13.3 & 14.4 & 22.2 \\
\hline \multicolumn{6}{|l|}{ Uso de la violencia } \\
\hline 14. Si un niño pega a tu hijo, éste debe responderle de la misma forma & 15.0 & 77.2 & 7.8 & 5.0 & 10.0 \\
\hline $\begin{array}{l}\text { 15. Los profesores de la escuela hacen bien en utilizar el castigo físico contra niños que son } \\
\text { repetidamente desobedientes y rebeldes }\end{array}$ & 8.3 & 88.3 & 3.3 & 4.4 & 3.9 \\
\hline $\begin{array}{l}\text { 16. Los niños, realmente, no se dan cuenta de que sus padres pegan a sus madres a no ser } \\
\text { que sean testigos de una pelea }\end{array}$ & 28.3 & 64.4 & 7.2 & 10.0 & 18.3 \\
\hline 17. Las bofetadas son a veces necesarias & 17.8 & 75.0 & 7.2 & 8.3 & 9.4 \\
\hline 18. Para maltratar a una mujer hay que odiarla & 11.1 & 79.4 & 9.4 & 3.9 & 7.2 \\
\hline $\begin{array}{l}\text { 19. La mayoría de los hombres que agreden a sus parejas creen que su comportamiento está } \\
\text { justificado }\end{array}$ & 26.7 & 68.9 & 4.4 & 7.8 & 18.9 \\
\hline $\begin{array}{l}\text { 20. La mayoría de los hombres que agreden a sus parejas se sienten avergonzados y culpa- } \\
\text { bles por ello }\end{array}$ & 44.4 & 46.7 & 8.9 & 13.3 & 31.1 \\
\hline 21. Los golpes en el trasero (a un niño) son a veces necesarios & 46.7 & 43.9 & 9.4 & 22.8 & 23.9 \\
\hline 22. Lo que ocurre en una familia es problema únicamente de la familia & 46.1 & 42.8 & 11.1 & 10.6 & 35.6 \\
\hline 23. Muy pocas mujeres tienen secuelas físicas o psíquicas a causa de los malos tratos & 12.8 & 80 & 7.2 & 5 & 7.8 \\
\hline 24. Si muchas mujeres no fastidiaran tanto a sus maridos, seguramente no serían maltratadas & 33.6 & 55.6 & 8.9 & 14.4 & 21.1 \\
\hline 25. Las mayoría de los maltratadores son personas fracasadas o "perdedores" & 32.8 & 58.3 & 8.9 & 9.4 & 23.3 \\
\hline 26. Las mujeres a menudo lesionan también a sus maridos & 60.0 & 23.9 & 16.1 & 17.8 & 42.2 \\
\hline 27. Cuando tus vecinos se están pegando. es responsabilidad tuya intervenir & 62.8 & 30.6 & 6.7 & 20.6 & 42.2 \\
\hline 28. Siempre es un delito que un hombre pegue a una mujer & 89.4 & 8.3 & 2.2 & 4.4 & 85.0 \\
\hline $\begin{array}{l}\text { 29. Los agresores son personas con graves problemas psicológicos que a menudo no saben } \\
\text { lo que hacen }\end{array}$ & 48.3 & 41.7 & 10 & 14.4 & 33.9 \\
\hline
\end{tabular}

\section{Descripción de las puntuaciones}

En la tabla 2 se presentan las puntuaciones obtenidas con la corrección original del inventario (dos dimensiones, formato de respuesta verdadero o falso) y con la propuesta en 4 factores y respuesta Likert de Ferrer, Bosch, Ramis, Torres, et al. (2006). 
Tabla 2. Puntuaciones medias

\begin{tabular}{llrcc}
\hline & & \multicolumn{3}{c}{ Muestra Total } \\
& & $\mathrm{M}=180)$ \\
\hline & & 3.14 & 2.38 & $0-17$ \\
IPD-M $_{\text {(13 items) }}$ & & 5.86 & 2.4 & $0-13$ \\
IPD-V $_{\text {(16 items) }}$ & & 9.01 & 4.06 & $0-22$ \\
IPDMV $_{\text {(29items) }}$ & & 8.92 & 2.81 & $7-22$ \\
Factor 1 $^{*}$ & Total & 1.27 & 0.40 & $1-3.14$ \\
& Promerido & 17.72 & 6.12 & $8-59$ \\
Factor 2 & Total & 2.21 & 0.76 & $1-7.38$ \\
& Promerido & 8.89 & 3.35 & $5-18$ \\
Factor 3 & Total & 1.78 & 0.67 & $1-3.60$ \\
& Promerido & 7.99 & 2.79 & $4-16$ \\
Factor 4 & Total & 1.99 & 0.70 & $1-4$ \\
Total & Promerido & 43.52 & 10.52 & $24-90$ \\
\hline
\end{tabular}

Nota. Factor 1: estereotipos machistas; Factor 2: culpabilización de la mujer; Factor 3: aceptación de la violencia como forma de resolver conflictos; Factor 4: minimización de la violencia/ desculpabilización del agresor. Total = puntuación total 24 ítems con respuesta Likert de Ferrer et al. (2006).

\section{Fiabilidad}

Para valorar la consistencia del inventario en sus opciones de corrección, se realizaron diversos análisis de fiabilidad alfa de Cronbach (ver tabla 3). La consistencia interna del inventario completo fue mayor para la versión factorial (respuesta
Likert) que para la original. Respecto a las escalas o factores, el factor cuatro $(\alpha=.374)$ y la escala sobre violencia $(\alpha=.519)$ presentaron las fiabilidades más bajas. Por último se analizó el total de los ítems (29) con formato de respuesta Likert, obteniéndose una fiabilidad superior al total de la versión con respuesta verdadero/falso ( $\alpha=.732$ vs. $\alpha=.718)$.

Tabla 3. Fiabilidad del inventario en sus distintas opciones de corrección

\begin{tabular}{|c|c|c|c|c|c|c|c|c|}
\hline \multicolumn{3}{|c|}{ Propuesta original } & \multicolumn{5}{|c|}{ Propuesta Ferrer et al. (2006) } & \multirow{2}{*}{ Likert $_{-29 \text { items }}$} \\
\hline IPDM & IPDV & IPDMV & F1 & $\mathrm{F} 2$ & F3 & F4 & Total & \\
\hline .662 & .519 & .718 & .658 & .644 & .601 & .374 & .758 & .732 \\
\hline
\end{tabular}

Nota. F1: estereotipos machistas; F2: culpabilización de la mujer; F3: aceptación de la violencia como forma de resolver conflictos; $\boldsymbol{F 4}$ : minimización de la violencia/ desculpabilización del agresor. Total = puntuación total 24 ítems con respuesta Likert de Ferrer et al. (2006).

\section{Sensibilidad al cambio terapéutico}

Junto a los descriptivos y la consistencia interna del inventario, se quiso poner a prueba su sensibilidad para detectar cambios terapéuticos, algo indicado por los autores de la herramienta. En la tabla 4 se describen las puntuaciones medias de 30 de los agresores, que realizaron el programa de tratamiento en prisión y a los que se evaluó antes y después del mismo. Los resultados con la corrección original impiden afirmar la existencia de cambios en la puntuación, poniendo de manifiesto la poca sensibilidad de la herramienta (se debe tener en cuenta que las diferencias en las puntuaciones pueden suponer tan solo una respuesta afirmativa más o menos, pues el nivel de posible distorsión es bajo aun incluyendo ítems con respuestas afirmativas en más del $60 \%$ de los casos). Por otro lado, la corrección de la propuesta factorial (respuesta Likert) detecta cambios en el factor 2 (culpabilizar a la mujer) y diferencias estadísticamente significativas en la puntuación total. 
Tabla 4. Puntuaciones pre y post tratamiento

\begin{tabular}{|c|c|c|c|c|c|c|c|}
\hline & \multicolumn{2}{|c|}{$\begin{array}{l}\text { Pre-tratamiento } \\
\qquad(\mathrm{N}=30)\end{array}$} & \multicolumn{2}{|c|}{$\begin{array}{l}\text { Post-tratamiento } \\
(\mathrm{N}=30)\end{array}$} & \multirow[b]{2}{*}{$t$} & \multirow[b]{2}{*}{$p$} & \multirow[b]{2}{*}{$d$} \\
\hline & $\mathrm{M}$ & (DT) & $\mathrm{M}$ & (DT) & & & \\
\hline IPD-M $_{(13 \text { items })}$ & 2.88 & $(2.40)$ & 2.54 & $(1.74)$ & .645 & .525 & .16 \\
\hline IPD-V & 5.58 & $(2.87)$ & 5.04 & $(2.11)$ & 1.182 & .249 & .21 \\
\hline IPDMV $_{\left(29_{\text {items })}\right.}$ & 8.46 & $(4.58)$ & 7.58 & $(2.75)$ & 1.027 & .315 & .23 \\
\hline Factor 1 & 1.52 & $(0.48)$ & 1.39 & $(0.46)$ & 1.466 & .156 & .28 \\
\hline Factor 2 & 2.23 & $(0.59)$ & 2.00 & $(0.45)$ & 2.118 & .045 & .43 \\
\hline Factor 3 & 1.75 & $(0.67)$ & 1.54 & $(0.43)$ & 1.867 & .075 & .37 \\
\hline Factor 4 & 2.30 & $(0.64)$ & 2.10 & $(0.57)$ & 1.453 & .160 & .33 \\
\hline Total $_{\text {(factorial) }}$ & 46.54 & (10.04) & 41.87 & $(7.40)$ & 2.802 & .010 & .53 \\
\hline
\end{tabular}

Nota. Factor 1: estereotipos machistas; Factor 2: culpabilización de la mujer; Factor 3: aceptación de la violencia como forma de resolver conflictos; Factor 4: minimización de la violencia/ desculpabilización del agresor. Total = puntuación total 24 ítems con respuesta Likert de Ferrer et al. (2006).

\section{Estructura factorial del inventario}

La medida de adecuación muestral de Kaiser-Meyer-Olkin superior a $0.5(\mathrm{KMO}=.706)$ y la prueba de esfericidad de Bartlett significativa $(p=.000)$ confirman la adecuación de aplicar el análisis factorial. En un primer momento se analizó la estructura factorial del inventario completo (29 ítems). El análisis de comunalidades y del gráfico de sedimentación indicó como mejor solución la organización en cuatro factores. Se optó por suprimir aquellos ítems con coeficientes inferiores a .4 (Stevens, 2009; Tabachnick y Fidell, 2007), incluyendo solo aquellos con coeficientes superiores a .3 que incrementasen la consistencia interna del factor.

Este primer análisis puso de manifiesto que los ítems $7,8,19$ y 28 podían ser eliminados por sus bajas cargas factoriales o poco aporte a la consistencia de la escala. Junto a estos, el ítem 27 fue eliminado del análisis final por considerarlo improcedente (ver discusión). Tras la eliminación de los ítems, el conjunto del inventario presentó un consistencia interna media/alta $(\alpha=.759)$. Los factores 1,2 y 3 presentaron una consistencia media $(\alpha>$ .6) y el 4 baja $(\alpha=.496)$. 
Tabla 5. Matriz de componentes rotados

\begin{tabular}{|c|c|c|c|c|}
\hline \multirow[b]{2}{*}{ Ítem } & \multicolumn{4}{|c|}{ Componente } \\
\hline & $\begin{array}{c}1 \\
\text { Estereotipos } \\
\text { machistas }\end{array}$ & $\begin{array}{c}2 \\
\text { Culpabilización } \\
\text { de la mujer }\end{array}$ & $\begin{array}{c}3 \\
\text { Aceptación } \\
\text { de la violencia }\end{array}$ & $\begin{array}{c}4 \\
\begin{array}{c}\text { Desculpabilización } \\
\text { del agresor }\end{array}\end{array}$ \\
\hline 1 & .421 & & & \\
\hline 2 & .620 & & & \\
\hline 3 & .601 & & & \\
\hline 4 & .777 & & & \\
\hline 5 & .547 & & & \\
\hline 6 & .479 & & & \\
\hline 9 & & .379 & & \\
\hline 10 & & .579 & & \\
\hline 11 & & .683 & & \\
\hline 12 & .431 & & & \\
\hline 13 & & .417 & & \\
\hline 14 & & & .544 & \\
\hline 15 & & & .596 & \\
\hline 16 & & & & .500 \\
\hline 17 & & & .742 & \\
\hline 18 & & & & .441 \\
\hline 20 & & & & .366 \\
\hline 21 & & & .628 & \\
\hline 22 & & .459 & & \\
\hline 23 & & & & .396 \\
\hline 24 & & .619 & & \\
\hline 25 & & & & .547 \\
\hline 26 & & .576 & & \\
\hline 29 & & & & .694 \\
\hline Valores propios & 2.71 & 2.71 & 2.45 & 2.09 \\
\hline $\begin{array}{l}\text { Varianza explicada } \\
\text { (total } 36.87 \% \text { ) }\end{array}$ & $10.02 \%$ & $10.03 \%$ & $9.09 \%$ & $7.73 \%$ \\
\hline$\alpha$ Cronbach & .658 & .692 & .631 & .496 \\
\hline
\end{tabular}

Nota. Método de extracción: Análisis de componentes principales. Método de rotación: Normalización Varimax con Kaiser. La rotación ha convergido en 6 iteraciones.

\section{Análisis de estudios previos}

Como último objetivo, se quiso analizar los resultados disponibles de la aplicación del inventario en estudios previos. En la tabla 6 se presenta un resumen de 11 trabajos en los que se ha podido acceder a las puntuaciones en la herramienta, calculando el tamaño del efecto en el caso de existencia de diferencias entre grupos. 


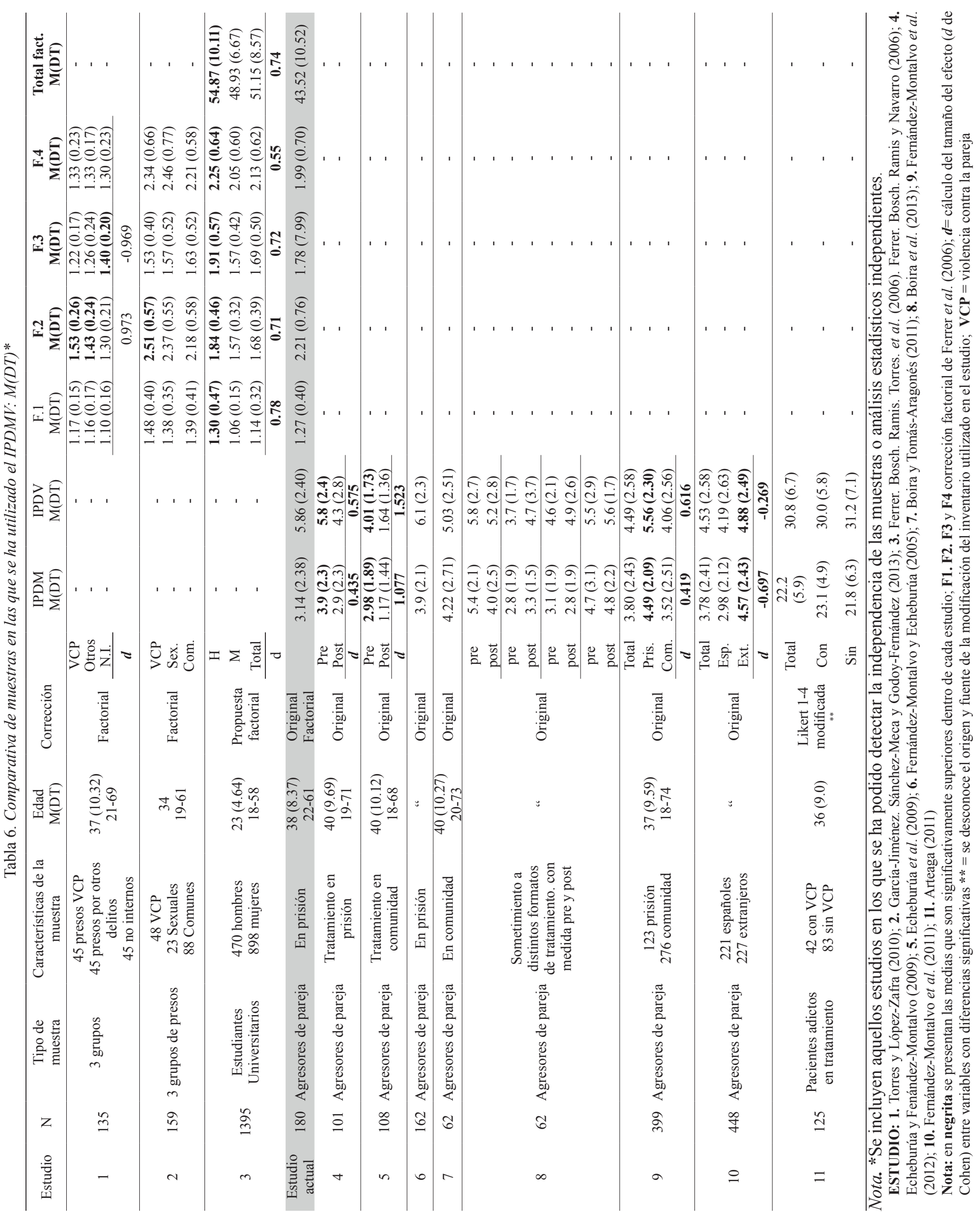


Los resultados han sido dispares respecto a la validez discriminante. La versión factorial del inventario no ha sido eficaz para diferenciar delincuentes y población general (estudio 1), con puntuaciones mayores en agresores en el factor $2(d=0.973)$, pero menores en el factor $3(d=-0.969)$ y sin diferencias en el resto de factores. Tampoco se han encontrado diferencias entre agresores de pareja, delincuentes sexuales y delincuentes comunes (estudio 2). Sin embargo, se ha mostrado sensible al género entre estudiantes (estudio $3)$, con puntuaciones mayores en hombres $(d=>0.7$ en los factores 1,2 y $3 ; d=0.55$ en el 4 ).

Con la corrección original, la herramienta ha diferenciado entre agresores en prisión o en comunidad (estudio 9), con puntuaciones mayores en los primeros (IPDM $d=0.419$ e IPDV $d=0.616$ ), y agresores españoles o extranjeros (estudio 10), con puntuaciones mayores en extranjeros (IPDM $d=0.697$ y IPDV $d=0.269$ ). Pese a ello, una versión modificada (estudio 11) no ha sido útil para diferenciar pacientes en tratamiento por adicciones con y sin violencia contra la pareja.

Los resultados disponibles sobre la sensibilidad al cambio terapéutico son positivos en trabajos de los autores de la herramienta, con tamaños del efecto pequeños para agresores en tratamiento en prisión (estudio 4: IPDM $d=0.435$ y IPDV $d=0.575$ ) y grandes para agresores en libertad (estudio 5: $\operatorname{IPDM} d=1.077$ y IPDV $d=1.523$ ). Otro trabajo (estudio 8), sin embargo, no ha confirmado esta sensibilidad.

\section{Discusión}

El presente estudio ha analizado las propiedades psicométricas de una escala diseñada para la evaluación de pensamientos distorsionados en agresores de pareja. Junto a ello, se ha comparado su funcionamiento en distintos estudios realizados desde su creación. El objetivo del trabajo se considera de especial relevancia por las implicaciones que se atribuyen a la variable, tanto para el mantenimiento de la violencia como para la evolución en programas de tratamiento (Lila, Herrero y Gracia, 2008b).

Debido a esta relevancia desde el punto de vista terapéutico, entre los objetivos del presente estudio se quiso analizar la sensibilidad del instrumento al cambio en los tratamientos, una utilidad indicada por sus creadores (Echeburúa y Fenández-Montalvo, 2009). Utilizando la corrección original, no pudo comprobarse un cambio significativo entre las evaluaciones pre y post tratamiento en la muestra de agresores. Sin embargo, se constataron diferencias significativas en el factor 2 y la puntuación total según la propuesta de Ferrer, Bosch, Ramis, Torres, et al. (2006). Estudios recientes tampoco han confirmado la mejora o reducción de las distorsiones autoinformadas con el tratamiento (Boira, López, Tomás-Aragonés y Gaspar, 2013; Martínez y Pérez, 2009).

Una hipótesis derivada de la aplicación del IPDMV era la existencia de ítems poco discriminativos, a los que cualquier persona podría responder afirmativamente sin necesidad de presentar distorsiones cognitivas. El análisis de frecuencias puso de manifiesto la existencia de ítems muy prevalentes, entre los que se encontraban dos que se considera deberían ser eliminados. Al margen de los eliminables por los criterios estadísticos propios del análisis factorial, que a continuación analizaremos, desde el punto de vista teórico se propondría la eliminación del ítem 27 ("Cuando tus vecinos se están pegando, es responsabilidad tuya intervenir") y el ítem 28 ("Siempre es un delito que un hombre pegue a una mujer"). Su consideración como distorsiones cognitivas puede entenderse un error para el que habría tres posibles explicaciones: 1) su redacción es errónea; 2) se trata de ítems inversos que no han sido señalados como tales en las instrucciones del inventario; o 3) contienen matices semánticos que deberían quedar al margen del ámbito de las distorsiones (por ejemplo: ¿la responsabilidad no es de intervenir sino de ayudar o informar? o ¿no es responsabilidad sino otra cosa?; ¿no es un delito en caso de que el hombre pegue en defensa propia?). Algo menos evidente sería el caso del ítem 19 ("La mayoría de los hombres que agreden a sus parejas creen que su comportamiento está justificado"), cuyo contenido hace mención precisamente al eje central del trabajo con las distorsiones en agresores, esto es, la justificación de lo ocurrido.

Los criterios estadísticos aplicados en el análisis factorial (cargas factoriales $\geq .40 ; 0 \geq .30$ si mejoraban el $\alpha$ de Cronbach), permitieron reducir el número total de ítems, eliminando los ítems 7, 8, 19 y 28 por carecer de significación estadística. Junto al criterio estadístico, se decidió eliminar el ítem 27 siguiendo el criterio antes mencionado. El análisis factorial final agrupó el contenido del inventario en cuatro factores con la siguiente composición: 1) Estereotipos machistas: ítems 1, 2, 3, 4, 5, 6 y $12(\alpha=.658)$; 2) Culpabilización de la mujer: ítems 9, 10, 11, 13, 22, 24 y $26(\alpha=.692)$; 3) Aceptación de la violencia: ítems 14, 15, 17 y $21(\alpha=.631)$; y 4$)$ Desculpabilización del agresor: ítems $16,18,20,23,25$ y $29(\alpha=.496)$.

$\mathrm{El}$ análisis de la fiabilidad del inventario y sus factores, ha puesto de manifiesto que las opciones de corrección factoriales y con opción de respuesta tipo Likert son más 
consistentes que la propuesta original. Recordemos que el alfa de Cronbach aumenta conforme se incrementa el número de ítems, y esas versiones contienen menos ítems que la versión original. La consistencia total del inventario ascendió a $\alpha=.759$ con la eliminación de los ítems y la de los factores propuestos en este trabajo mejoró la obtenida al aplicar la propuesta de Ferrer, Bosch, Ramis, Torres, et al. (2006) en esta misma muestra. Sin embargo, los valores obtenidos son cuestionables, especialmente malos para el factor 4 ("desculpabilización del agresor"). Todos los valores obtenidos han sido inferiores a los indicados por los autores del inventario.

Los factores encontrados coincidieron en gran medida con los de la propuesta de Ferrer, Bosch, Ramis, Torres, et al. (2006), en especial el factor 1, aunque se considera que la coherencia temática de los mismos ha resultado ser superior en el presente trabajo, posiblemente debido a la muestra utilizada (agresores condenados por violencia contra la pareja frente a estudiantes universitarios). La necesidad de eliminar ítems y la posibilidad de reorganizar el contenido ha puesto de manifiesto las limitaciones de la propuesta original. Junto al análisis factorial, del análisis psicométrico del inventario se desprende que tanto la fiabilidad como la sensibilidad al cambio son más precisas con la opción de respuesta tipo Likert y solución factorial.

Respecto a la revisión de estudios previos, se ha indicado la ausencia de cambios significativos tras la intervención terapéutica (Boira et al., 2013). Esto podría deberse a la ausencia de modificación en los grupos tratados o a la ausencia de sensibilidad de la escala (una cuestión a resolver en futuros trabajos). Por otro lado, no se han encontrado muchas diferencias entre agresores de pareja y la población general (Torres y López-Zafra, 2010), ni entre agresores de pareja, delincuentes sexuales y delincuentes comunes (García-Jiménez et al., 2013). La escala tampoco ha permitido diferenciar sujetos con y sin VCP entre pacientes adictos en tratamiento (Arteaga, 2011).

Junto a los trabajos analizados, en otra línea de investigación el inventario tuvo que ser eliminado del protocolo de evaluación utilizado para la creación de una herramienta para valorar el tratamiento en la comunidad por no encontrar saturaciones factoriales significativas (Pérez y Martínez, 2011) y tampoco mostró sensibilidad al cambio terapéutico (Martínez y Pérez, 2009), aunque en estos casos no se pudo acceder a las puntuaciones de la herramienta.

Todos estos resultados pondrían de manifiesto la necesidad de utilizar el inventario con cautela en caso de ser un criterio terapéutico, o de riesgo, y la necesidad de acumular más resultados sobre sus propiedades psicométricas antes de afirmar su posible utilidad.

Desde la experiencia práctica, Murphy y Eckhardt (2005) señalan que las distorsiones son constructos no accesibles al sujeto en ausencia de indicadores (precipitantes) internos o externos y, por ello, no se debe presuponer que los agresores tendrán acceso directo a ellas simplemente por acudir a tratamiento o solicitar su autoinforme con un cuestionario como el utilizado en este estudio. Asimismo, al igual que ocurre con otras variables como las afectivas (Loinaz, Echeburúa y Ullate, 2012), las distorsiones no son constructos unidimensionales (Peters, 2008) y pueden hacer referencia a distintos aspectos, para distintos contextos y bajo distintas circunstancias. Por ejemplo, la minimización y la negación podrían abarcar la existencia de violencia, su gravedad, la responsabilidad sobre lo ocurrido o la necesidad de intervención. A su vez, podrían relacionarse solo con una víctima concreta y no ser generalizables a otros aspectos. Estas particularidades deberían ser consideradas en el ámbito de estudio.

Este trabajo presenta una serie de limitaciones que podrían ser solventadas en próximos estudios. Por un lado, no se ha comparado la muestra actual con un grupo de control. Por ello, resultará necesario analizar la validez discriminante de la propuesta factorial, comparando las puntuaciones de los agresores con las de un grupo de control proveniente de la población general. En la revisión bibliográfica se ha puesto de manifiesto cómo las distorsiones y estereotipos están presentes en la población general (Ferrer, Bosch, Ramis, Torres et al., 2006; Valor-Segura et al., 2008, 2011), pero son más prevalentes entre los agresores (Gilchrist, 2007, 2009) y, de hecho, son el objetivo de los tratamientos afirmando que su mejora es signo de eficacia (Carbajosa, Boira y TomásAragonés, 2013; Lila et al., 2013; Millana, 2011). Por ello, es imprescindible demostrar la validez de los instrumentos y la existencia de diferencias en las puntuaciones para poder tomarlos como referencia. La comparación en el presente trabajo de los estudios disponibles con el IPDMV no ha permitido sacar conclusiones al respecto debido a la poca concordancia entre estudios.

Por otro lado, no se ha correlacionado el autoinforme en distorsiones con ningún otro criterio ni se ha controlado la deseabilidad social. Podrá resultar de interés analizar la validez convergente de la herramienta. Bien comparando su capacidad con la de otros instrumentos con objetivos similares, como el Cuestionario de Creencias Irracionales (Arce y Fariña, 2005), la Escala de Atribución de responsabilidad y Minimización (Lila et al., 2008a,b), o la Escala de 
Cultura de Honor (López-Zafra, 2007). O bien, tratando de correlacionar el nivel de distorsión con el tipo de delito o el autoinforme de violencia en escalas como la Conflict Tactics Scales-2 (Straus, Hamby, Boney-McCoy y Sugarman, 1996).

Por último, tal vez sea necesario proponer un nuevo instrumento con el mismo objetivo pero que abarque mejor los estilos de distorsión presentes en muestras de agresores (aquellas que se puedan considerar criminógenas). Para ello, podrá resultar de interés aplicar la metodología utilizada por otros estudios (Lila et al., 2008a,b; Peters, 2008; Scott y Straus, 2007) y analizar de forma cualitativa las narraciones que los agresores realizan en el momento actual, tras reformas legales y sociales que facilitan la utilización de machismos más sutiles (Valor-Segura et al., 2011) o atribuciones de culpa más allá de la víctima (al sistema judicial o a los movimientos feministas, por ejemplo).

Las implicaciones de los análisis psicométricos van más allá de su interés descriptivo. La utilización de herramientas adecuadas, válidas y fiables, es imprescindible para poder valorar la utilidad y eficacia de los programas de intervención, ver sobre qué variables tienen efecto y, por último, tener en cuenta qué variables de los agresores influyen sobre el riesgo de reincidencia (Loinaz, 2014) o sobre el desistimiento en la VCP (Walker, Bowen y Brown, 2013).

\section{Referencias}

Arce, R., y Fariña, F. (2005). Cuestionario de creencias irracionales y pensamientos distorsionados sobre el empleo de la violencia, los roles de género y la relación de pareja (dependencia emocional). Manuscrito no publicado. Unidad de Psicología Forense: Universidad de Santiago de Compostela.

Arteaga, A. (2011). Prevalencia de violencia contra la pareja en pacientes adictos en tratamiento: diferencias en el perfil de pacientes con y $\sin$ conductas violentas. Trabajo fin de máster, Universidad Pública de Navarra, Pamplona.

Beech, A. R., Craig, L. A., y Browne, K. D. (Eds.) (2009). Assessment and treatment of sex offenders: A handbook. Chichester, UK: WileyBlackwell.

Boer, D. P., Eher, R., Craig, L. A., Miner, M. H., y Pfäfflin, F. (Eds.) (2011). International perspectives on the assessment and treatment of sexual offenders: Theory, practice, and research. Chichester, UK: John Wiley \& Sons.

Boira, S., López, Y., Tomás-Aragonés, L., y Gaspar, A. R. (2013). Intervención psicológica en la comunidad en hombres condenados por violencia de género. Anales de Pisología, 29, 19-28. doi: 10.6018/ analesps.29.1.130631

Boira, S., y Tomás-Aragonés, L. (2011). Características psicológicas y motivación para el cambio en hombres condenados por violencia contra la pareja. International Journal of Psychological Research, 4, 48-56.

Bowen, E. (2011). The rehabilitation of partner-violent men. Chichester, UK: Wiley-Blackwell.

Bowen, E., y Gilchrist, E. (2004). Do court- and self-referred domestic violence offenders share the same characteristics? A preliminary comparison of motivation to change, locus of control and anger. Legal and Criminological Psychology, 9, 279-294. doi: 10.1348/1355325041719383
Carbajosa, P., Boira, S., y Tomás-Aragonés, L. (2013). Difficulties, skills and therapy strategies in interventions with court-ordered batterers in Spain. Aggression and Violent Behavior, 18, 118-124. doi: 10.1016/j. avb.2012.11.005

Dobash, E. R., y Dobash, R. P. (2011). What were they thinking? Men who murder an intimate partner. Violence against Women, 17, 111-134. doi: 10.1177/1077801210391219

Dutton, D. G. (1986). Wife assaulter's explanations for assault: The neutralization of self-punishment. Canadian Journal of Behavioural Science/Revue canadienne des sciences du comportement, 18, 381-390.

Dutton, D. G., y Starzomski, A. J. (1994). Psychological differences between court-referred and self-referred wife assaulters. Criminal Justice and Behavior, 21, 203-222. doi: 10.1177/0093854894021002002

Eckhardt, C. I., y Kassinove, H. (1998). Articulated cognitive distortions and cognitive deficiencies in maritally violent men. Journal of Cognitive Psychotherapy, 12, 231-250.

Echeburúa, E., y Fenández-Montalvo, J. (2009). Evaluación de un programa de tratamiento en prisión de hombres condenados por violencia grave contra la pareja. International Journal of Clinical and Health Psychology, 9, 5-20.

Echeburúa, E., y Fernández-Montalvo, J. (1998). Hombres maltratadores. En E. Echeburúa y P. Corral (Eds.), Manual de violencia familiar (pp. 112-113). Madrid: : Siglo XXI.

Echeburúa, E., Sarasua, B., Zubizarreta, I., y de Corral, P. (2009). Evaluación de la eficacia de un tratamiento cognitivo-conductual para hombres violentos contra la pareja en un marco comunitario: una experiencia de 10 años (1997-2007). International Journal of Clinical and Health Psychology, 9, 199-217.

Fernández-Montalvo, J., Echauri, J. A., Martínez, M., y Azcárate, J. M. (2012). Batterer men in prison and in court-referred treatment programmes: What is the difference? Spanish Journal of Psychology, 15, 315-322.

Fernández-Montalvo, J., Echauri, J.A., Matínez, M., y Azcárate, J.M. (2011). Violencia de género e inmigración: perfil diferencial de hombre maltratadores nacionales e inmigrantes. Psicología Conductual/ Behavioral Psychology, 19, 439-452.

Fernández-Montalvo, J., y Echeburúa, E. (2005). Hombres condenados por violencia grave contra la pareja: un estudio psicopatológico. Análisis y Modificación de Conducta, 31, 451-475.

Ferrer, V. A., Bosch, E., Ramis, M. C., Torres, E. G., y Navarro, C. (2006). La violencia contra las mujeres en la pareja: creencias y actitudes en estudiantes universitarios. Psicothema, 18, 359-366.

Ferrer, V. A., Bosch, E., Ramis, M. C., y Navarro, C. (2006). Las creencias y actitudes sobre la violencia contra las mujeres en la pareja: determinantes sociodemográficos, familiares y formativos. Anales de Pisología, 22, 251-259.

Gannon, T. A., Ward, T., Beech, A. R., y Fisher, D. (Eds.). (2007). Aggressive Offenders' Cognition: Theory, research, and practice. Chichester, UK: John Wiley \& Sons.

García-Jiménez, J. G., Sánchez-Meca, J., y Godoy-Fernández, C. (2013). Distorsiones cognitivas respecto a la violencia de género en presos. En F. Expósito, I. Valor-Segura, M. Vilariño y A. Palmer (Eds.), Psicología Jurídica aplicada a los problemas sociales (pp. 89-96). Santiago de Compostela: Sociedad Española de Psicología Jurídica y Forense.

Gilchrist, E. (2007). The Cognition of Domestic Abusers: Explanations, Evidence and Treatment. En T.A. Gannon, T. Ward, A.R. Beech y D. Fisher (Eds.), Aggressive Offenders' Cognition: Theory, research, and practice (pp. 247-266). Chichester, UK: John Wiley \& Sons.

Gilchrist, E. (2009). Implicit thinking about implicit theories in intimate partner violence. Psychology, Crime \& Law, 15, 131-145. doi: $10.1080 / 10683160802190863$

Helmus, L., Hanson, R. K., Babchishin, K. M., y Mann, R. E. (2013). Attitudes supportive of sexual offending predict recidivism: A meta-analysis. Trauma, Violence, \& Abuse, 14, 34-53. doi: 10.1177/1524838012462244

Henderson, M., y Hewstone, M. R. (1984). Prison inmates' explanations for interpersonal violence: Accounts and attributions. Journal of Consulting and Clinical Psychology, 52, 789-794. 
Henning, K., y Holdford, R. (2006). Minimization, denial, and victim blaming by batterers: How much does the truth matter? Criminal Justice and Behavior, 33, 110-130. doi: 10.1177/0093854805282322

Henning, K., Jones, A., y Holdford, R. (2005). “I didn’t do it, but if I did I had a good reason": Minimization, denial, and attributions of blame among male and female domestic violence offenders. Journal of Family Violence, 20, 131-139. doi: 10.1007/s10896-005-3647-8

Holtzworth-Munroe, A., y Hutchinson, G. (1993). Attributing negative intent to wife behavior: The attributions of maritally violent versus nonviolent men. The Journal of Abnormal Psychology, 102, 206-211.

Lila, M., Gracia, E., y Herrero, J. (2012). Asunción de responsabilidad en hombres maltratadores: influencia de la autoestima, la personalidad narcisista y la personalidad antisocial. Revista Latinoamericana de Psicología, 44, 99-108.

Lila, M., Herrero, J., y Gracia, E. (2008a). Atribución de responsabilidad y minimización en hombres penados por violencia contra la mujer: un instrumento de evaluación. En F. J. Rodríguez, C. Bringas, F. Fariña, R. Arce y A. B. Bernardo (Eds.), Psicología Jurídica: familia y victimología (pp. 271-279). Oviedo: Universidad de Oviedo.

Lila, M., Herrero, J., y Gracia, E. (2008b). Evaluating attribution of responsibility and minimization by male batterers: Implications for batterer programs. The Open Criminology Journal, 1, 4-11.

Lila, M., Oliver, A., Galiana, L., y Gracia, E. (2013). Predicting success indicators of an intervention programme for convicted intimate-partner violence offenders: the Contexto Programme. The European Journal of Psychology Applied to Legal Context, 5, 73-95.

Loinaz, I. (2014). Typologies, risk and recidivism in partner-violent men with the B-SAFER: a pilot study. Psychology, Crime \& Law, 20(2), 183-198. doi: 10.1080/1068316x.2013.770854

Loinaz, I., Echeburúa, E., y Ullate, M. (2012). Estilo de apego, empatía y autoestima en agresores de pareja. Terapia Psicológica, 30, 61-70.

Loinaz, I., Ortiz-Tallo, M., y Ferragut, M. (2012). MCMI-III grossman personality facets among partner-violent men in prison. International Journal of Clinical and Health Psychology, 12, 389-404.

Loinaz, I., Ortiz-Tallo, M., Sánchez, L. M., y Ferragut, M. (2011). Clasificación multiaxial de agresores de pareja en centros penitenciarios. International Journal of Clinical and Health Psychology, 11, 249-268.

López-Zafra, E. (2007). Elaboracion de una escala para medir Cultura del Honor. Revista de Psicología Social, 22, 31-42. doi: 10.1174/021347407779697520

Martínez, M., y Pérez, M. (2009). Evaluación criminológica y psicológica de los agresores domésticos. Invesbreu, 46, 11-16.

Maruna, S., y Mann, R. E. (2006). A fundamental attribution error? Rethinking cognitive distortions. Legal and Criminological Psychology, 11, 155-177. doi: 10.1348/135532506x114608

Millana, L. (2011). Intervention programs for Spanish inmate aggressors convicted of domestic violence. The Open Criminology Journal, 4, 91-101.

Murphy, C. M., y Eckhardt, P. C. I. (2005). Treating the abusive partner: An individualized cognitive-behavioral approach. New York, NY: The Guilford Press.

Novo, M., Fariña, F., Seijo, M. D., y Arce, R. (2012). Assessment of a community rehabilitation programme in convicted male intimate-partner violence offenders. International Journal of Clinical and Health Psychology, 12, 219-234.

Ó Ciardha, C., y Ward, T. (2013). Theories of cognitive distortions in sexual offending: What the current research tells us. Trauma, Violence, \& Abuse, 14, 5-21. doi: 10.1177/1524838012467856

Pérez, M., y Martínez, M. (2011). Evaluación de los programas formativos aplicados desde la ejecución penal en la comunidad para delitos de violencia de género. En Intervención con agresores de violencia de género, (pp. 13-150) Barcelona: Centro de Estudios Jurídicos y Formación Especializada.

Peters, J. (2008). Measuring myths about domestic violence: Development and initial validation of the domestic violence myth acceptance scale. Journal of Aggression, Maltreatment \& Trauma, 16, 1-21. doi: 10.1080/10926770801917780

Rathus, J. H., y Feindler, E. L. (2004). Assessment of partner violence: A handbook for researchers and practitioners. Washington, DC: American Psychological Association.

Russell, M. N. (2002). Changing beliefs of spouse abusers. En J. McGuire (Ed.), Offender rehabilitation and treatment: Effective programmes and policies to reduce re-offending (pp. 243-258). Chichester, UK: John Wiley \& Sons.

Scott, K., y Straus, M. (2007). Denial, minimization, partner blaming, and intimate aggression in dating partners. Journal of Interpersonal Violence, 22, 851-871. doi: 10.1177/0886260507301227

Shaver, P. R., y Mikulincer, M. (Eds.). (2011). Human aggression and violence: Causes, manifestations, and consequences. Washington, DC: American Psychological Association.

Stevens, J. P. (2009). Applied multivariate statistics for the social sciences ( $5^{\mathrm{a}}$ ed.). New York, NY: Routledge.

Straus, M. A., Hamby, S., Boney-McCoy, S., y Sugarman, D. (1996). The Revised Conflict Tactics Scales: Development and preliminary psychometric data. Journal of Family Issues, 17, 283-316.

Tabachnick, B. G., y Fidell, L. S. (2007). Using multivariate statistics (5 ed.). Boston, MA: Pearson.

Torres, E., y López-Zafra, E. (2010). Diferencias en cultura del honor, inteligencia emocional y pensamientos distorsionados sobre las mujeres en reclusos y no reclusos. Boletín de psicología, 100, 71-88.

Trujano, P., y Raich, R. M. (2000). Variables socioculturales en la atribución de culpa a las víctimas de violación. Psicothema 12, 223-228.

Valor-Segura, I., Expósito, F., y Moya, M. (2008). Atribucion del comportamiento del agresor y consejo a la victima en un caso de violencia domestica. Revista de Psicología Social, 23, 171-180. doi: $10.1174 / 021347408784135896$

Valor-Segura, I., Expósito, F., y Moya, M. (2011). Victim blaming and exoneration of the perpetrator in domestic violence: The role of beliefs in a just world and ambivalent sexism. The Spanish Journal of Psychology, 14, 195-206. doi: 10.1177/1077801206291663

Walker, K., Bowen, E., y Brown, S. (2013). Desistance from intimate partner violence: A critical review. Aggression and Violent Behavior, 18, 271-280. doi: 10.1016/j.avb.2012.11.019

Wallach, H., y Sela, T. (2008). The importance of male batters' attributions in understanding and preventing domestic violence. Journal of Family Violence, 23, 655-660. doi: 10.1007/s10896-008-9189-0 